\title{
CORRELATION BETWEEN LOCUS POLYMORPHISM OF COL11A1 GENE AND OCULAR BIOMETRY IN ACUTE AND CHRONIC PRIMARY ANGLE CLOSURE GLAUCOMA
}

\author{
FITRATUL ILAHI ${ }^{1 *}$, ANDIKA PRAHASTA ${ }^{2}$, RIKA SUSANTI ${ }^{3}$, JAMSARI JAMSARI ${ }^{4}$ \\ ${ }^{1}$ Department of Ophthalmology, Biomedical Doctoral Program, Medical Faculty, Andalas University, Padang, West Sumatra, Indonesia. \\ ${ }^{2}$ Department of Ophthalmology, Medical Faculty, Padjadjaran University, Bandung, West Java, Indonesia. ${ }^{3}$ Department of Forensic Medical \\ Science, Medical Faculty, Andalas University, Padang, West Sumatra, Indonesia. ${ }^{4}$ Department of Agriculture, Faculty of Agriculture. Andalas \\ University, Padang, West Sumatra, Indonesia. Email: fitratulilahispm@gmail.com
}

Received: 22 January 2021, Revised and Accepted: 09 March 2021

\section{ABSTRACT}

Objective: The objective of this study is to analyze the correlation between locus polymorphisms of genetics variants of rs3753841 (COL11A1) and ocular biometry, including anterior chamber depth (ACD), axial length (AL), and lens thickness (LT) in acute and chronic primary angle-closure glaucoma (PACG) patients.

Methods: A total of 50 patients consisting of 25 acute PACG and 25 chronic PACG patients were included in this study. Ocular biometric parameters including anterior ACD, AL, and LT measurement were performed in all subjects using A-scan biometry. DNA isolation from blood samples was performed using PCR sequencing for genotyping locus polymorphisms of rs3753841 (COL11A1).

Results: Mean ACD was shallower (2.40 $\pm 0.40 \mathrm{~mm})$, mean AL was shorter (21.86 $\pm 1.31 \mathrm{~mm})$, and mean LT was thicker $(5.01 \pm 0.49 \mathrm{~mm})$ in acute PACG compared to chronic PACG group, yet with no significant difference ( $p>0.05$ ). We found a statistically significant difference of rs3753841 (COL11A1) polymorphism, with heterozygous mutant was mostly found in chronic PACG and homozygous mutant was mostly found in acute PACG patients $(\mathrm{p}<0.05)$. Significant correlations were also found between the locus polymorphism and ocular biometry (ACD and AL) in chronic PACG ( $<<0.05)$.

Conclusion: We confirmed the polymorphisms of rs3753841 (COL11A1) in both groups and its significant correlation with ocular biometry (ACD and AL) in chronic PACG.

Keywords: Primary angle-closure glaucoma, Anterior chamber depth, Axial length, Lens thickness, rs3753841 (COL11A1).

(C) 2021 The Authors. Published by Innovare Academic Sciences Pvt Ltd. This is an open access article under the CC BY license (http://creativecommons.org/ licenses/by/4.0/) DOI: http://dx.doi.org/10.22159/ajpcr.2021v14i5.40856. Journal homepage: https://innovareacademics.in/journals/index.php/ajpcr

\section{INTRODUCTION}

Primary angle-closure glaucoma (PACG) is one of the leading causes of irreversible blindness worldwide. Primary open-angle glaucoma (POAG) was known to be more frequent than PACG, but it is estimated that PACG can cause blindness five times more than POAG [1,2]. Early detection of PACG by effective screening and appropriate prophylaxis may help prevent blindness [3]

Angle-closure has been proved to be related with ocular biometric parameters, such as shallow anterior chamber depth (ACD), short axial length (AL), and thick crystalline lens. Shallow ACD $(<2.5 \mathrm{~mm})$ predisposes to PACG. Various studies have found a correlation between $\mathrm{ACD}, \mathrm{AL}$, and lens thickness (LT) in PACG, but to our knowledge, there have been no studies comparing ocular biometry with subtypes of acute and chronic PACG.

Genome wide association study found genetic variants of rs3753841 (COL11A1) which is susceptible to PACG [4]. It was also supported by several other studies in different countries and ethnicities $[5,6]$. Polymorphism of COL11A1 gene was linked to unprogressive axial myopia, possibly caused by a distorted fibrilar collagen matrix in the sclera. Meanwhile, eyes with PACG are usually hypermetropic with shorter AL and "crowded" anterior segment. Therefore, the variant of the COL11A1 gene that leads to PACG might have a different expression compared to the opposite effect observed in eyes with myopia [4,7]. In this study, we aimed to assess polymorphisms of rs3753841 (COL11A1) and its correlation with ocular biometry (ACD, AL, and LT) in acute and chronic PACG.

\section{METHODS}

This study was an observational study with a cross-sectional comparative study design, taking place in Dr. M. Djamil General Hospital Padang, West Sumatra-Indonesia, from March to December 2019. We obtained approval from the ethics committee with registered number $346 / \mathrm{KEP} / \mathrm{FK} / 2019$. The subjects of this study were acute and chronic PACG patients who met the inclusion criteria. All patients were examined and diagnosed by an experienced glaucoma consultant. Patients with media opacities, history of ocular trauma, history of previous trabeculectomy, history of lens extraction, and other ocular surgeries were excluded from this study.

We enrolled 50 patients in this study which consisted of 25 acute PACG patients and 25 chronic PACG patients. All subjects underwent complete ophthalmology examination of the anterior and posterior segment. Contact (non-immersion) ultrasonic biometry was performed (Nidek Co. Ltd, Gamagori, Japan) to measure AL, ACD, and LT in all subjects. We included one eye which met the inclusion criteria and if both eyes had symmetrical condition, the right eye was chosen.

The ACD measurement was classified into three categories; shallow $(<2.91 \mathrm{~mm})$, normal depth $(2.91-3.24 \mathrm{~mm})$, and deep $(>3.24 \mathrm{~mm})$. The measurement of AL was divided into short $(<24 \mathrm{~mm})$, normal length (24-26 mm), and long ( $>26 \mathrm{~mm}$ ). Last, the measurement of LT was grouped into the thin lens $(<3.59 \mathrm{~mm})$, normal thickness lens (3.595.07), and thick lens $(>5,07)$.

DNA isolation was extracted from patient's blood samples and PCR sequencing was performed for genotyping polymorphisms 
rs3753841 (COL11A1). PCR was performed using primers, as shown in Table 1.

A final volume of $25 \mu \mathrm{L}$ of blood serum containing $100 \mathrm{ng}$ DNA templates, $12.5 \mathrm{uL}$ of GoTaq Green Master Mix Promega, and 0.4 $\mu \mathrm{M}$ of primers was used for PCR sequencing. PCR was performed with an initial denaturation at $95^{\circ} \mathrm{C}$ for $3 \mathrm{~min}$, then followed by 35 cycles of a series of processes including denaturation at $95^{\circ} \mathrm{C}$ for $30 \mathrm{~s}$, annealing at $63^{\circ} \mathrm{C}$ for $30 \mathrm{~s}$, and elongation at $72^{\circ} \mathrm{C}$ for $1 \mathrm{~min}$. The PCR sequencing process ended with the final elongation stage at $72^{\circ} \mathrm{C}$ for $5 \mathrm{~min}$. PCR products were observed with electrophoresis using 1.5\% agarose gel, colored with GelRed DNA then observed with GelDoc. The PCR products were measured at $844 \mathrm{bp}$

The remaining PCR products were sent to Singapore's $1^{\text {st }}$ BASE for sequencing. Sequencing data were analyzed using Geneious bioinformatics software. The sequencing data for each sample from the forward primer were combined with the sequencing data from the reverse primer. Furthermore, the data were compared to BLAST on the NCBI website (http://blast.ncbi.nlm.nih.gov/Blast.cgi).

Polymorphism of rs3753841 (COL11A1) and biometric parameters were statistically analyzed between both groups. Statistical analysis was performed using the Statistical Package for the Social Sciences software version 17 (SPSS v17, IBM Corp., New York, NY, USA). Categorical data were compared using the Chi-square test and numerical data were compared using t-test. $\mathrm{p}<0.05$ were considered as a significant difference. Mean values were reported with standard deviation $($ mean $\pm S D)$.

\section{RESULTS}

This study is part of a larger study in which the correlation between ocular biometric parameters and gene polymorphisms previously found in PACG are evaluated thoroughly. Based on gender, there was no significant difference between the 2 groups $(\mathrm{p}=0.48)$. Acute PACG patients were younger (58.04 \pm 8.70 years) compared to chronic PACG patients (61.64 \pm 9.17 years), but the difference was not statistically significant.

We found no significant difference in ocular biometric measurements in both groups, as shown in Table $2(\mathrm{p}=0.494)$. Nevertheless, mean ACD was slightly shallower $(2.40 \pm 0.40 \mathrm{~mm})$ in acute PACG compared to chronic PACG $(2.47 \pm 0.32 \mathrm{~mm})$. The mean AL was shorter in acute PACG $(21.86 \pm 1.31 \mathrm{~mm})$ compared to chronic PACG group $(22.71 \pm 1.54)$, yet with no significant difference $(\mathrm{p}=0.319)$. There was also no significant difference in LT measurement between the two groups ( $p=0.432)$, although its mean value was found to be thicker in acute PACG $(5.01 \pm 0.49 \mathrm{~mm})$ compared to chronic PACG $(4.91 \pm 0.42)$.

We classified and statistically compared each ocular biometric parameter into three categories. The measurement of ACD was grouped into shallow $(<2.91 \mathrm{~mm})$, normal depth $(2.91-3.24 \mathrm{~mm})$, and

Table 1: Primers for genotyping rs3753841 (COL11A1)

\begin{tabular}{lll}
\hline Primers & Nucleotide & PCR product size \\
\hline rs3753841 F & 5'-GGTCCCCTCTAATGTGGCAT-3' & $844 \mathrm{bp}$ \\
rs3753841 R & 5'-GGTACCTCTCACTCTCCGCA-3' & \\
\hline
\end{tabular}

Table 2: Mean values of biometric parameters in acute and chronic PACG

\begin{tabular}{llll}
\hline Biometric parameters & Acute PACG & Chronic PACG & p-value \\
\hline ACD $(\mathrm{mm})$ & $2.40 \pm 0.40$ & $2.47 \pm 0.32$ & 0.494 \\
AL $(\mathrm{mm})$ & $21.86 \pm 1.31$ & $22.71 \pm 1.54$ & 0.319 \\
LT $(\mathrm{mm})$ & $5.01 \pm 0.49$ & $4.91 \pm 0.42$ & 0.432 \\
\hline
\end{tabular}

ACD: Anterior chamber depth, AL: Axial length, LT: Lens thickness,

PACG: Primary angle-closure glaucoma deep $(>3.24 \mathrm{~mm})$. The values of AL was grouped into short $(<24 \mathrm{~mm})$, normal length (24-26 mm), and long ( $>26 \mathrm{~mm})$, while LT was grouped into thin $(<3.59 \mathrm{~mm})$, normal thickness $(3.59-5.07 \mathrm{~mm})$, and thick lens (>5.07 mm). Using Chi-square test, we compared all those categories, as shown in Table 3

Table 3 shows no significant differences in ocular biometric parameters between the 2 groups ( $p>0.05)$. Nevertheless, most of the patients in both groups had a shallow ACD $(<2.91 \mathrm{~mm})$. Furthermore, we classified ACD measurement more specifically into three groups; $<2.10 \mathrm{~mm}, 2.10-$ $2.49 \mathrm{~mm}$, and $2.50-2.90 \mathrm{~mm}$. Based on AL measurement, all patients in both groups had a short AL $(<24 \mathrm{~mm})$. Therefore, we also classified short AL measurement more specifically into 3 ranges; <21 mm, 21-22 $\mathrm{mm}$, and 23-24 $\mathrm{mm}$, as shown in Table 4

We found a statistically significant difference in a more specific classification of shallow AL measurement between the two groups PACG ( $p=0.033$ ), but no significant difference in the classification of shallow ACD measurement. Based on Table 4, in the acute PACG group, there were more patients with AL measurement of $<21 \mathrm{~mm}$, meanwhile AL measurements of chronic PACG patients were mostly within the range of 21-22 $\mathrm{mm}$. These results showed that AL might have an important role in the mechanism of the crowded anterior segment and the progression of PACG into acute or chronic stage.

Polymorphisms of rs3753841 (COL11A1) in both groups consisted of wild type (CC), homozygous mutant (TT), and heterozygous mutant (CT). Electrophoresis and DNA bands from the isolated gene are shown in Fig. 1.

In this study, we found a significant difference in rs3753841 (COL11A1) polymorphism in which homozygous mutant was the most common type of mutation in acute PACG ( $\mathrm{p}=0.009)$, meanwhile heterozygous mutant was the most common mutation in chronic PACG, as shown in Table 5.

Correlation between rs3753841 (COL11A1) polymorphisms and ocular biometry parameters was analyzed between the two groups. Table 6

Table 3: Comparison of ocular biometric parameters in acute and chronic PACG

\begin{tabular}{llll}
\hline Biometric parameters & Acute PACG & Chronic PACG & p-value \\
\hline ACD $(\mathrm{mm})$ & & & \\
$<2.91$ & 22 & 23 & 0.073 \\
$2.91-3.24$ & 1 & 2 & \\
$>3.24$ & 2 & 0 & \\
AL $(\mathrm{mm})$ & & & \\
$<24$ & 25 & 25 & \\
$24-26$ & 0 & 0 & \\
$>26$ & 0 & 0 & \\
LT $(\mathrm{mm})$ & & & \\
$<3.59$ & 0 & 0 & \\
$3.59-5.07$ & 14 & 16 & \\
$>5.07$ & 11 & 9 & \\
\hline
\end{tabular}

ACD: Anterior chamber depth, AL: Axial length, LT: Lens thickness,

PACG: Primary angle-closure glaucoma

Table 4: Comparison of ranges in shallow ACD and short AL

\begin{tabular}{llll}
\hline Biometric parameters & Acute PACG & Chronic PACG & p-value \\
\hline ACD $(\mathrm{mm})$ & & & \\
$<2.10$ & 3 & 7 & 0.296 \\
$2.10-2.49$ & 10 & 13 & \\
$2.50-2.90$ & 12 & 5 & \\
AL $(\mathrm{mm})$ & & & 0.033 \\
$<21$ & 21 & 3 & \\
$21-22$ & 4 & 18 & \\
$23-24$ & 0 & 4 & \\
\hline
\end{tabular}

ACD: Anterior chamber depth, AL: Axial length, PACG: Primary angle-closure glaucoma 
shows the correlation between polymorphisms and ocular biometry in the acute PACG group. Based on Table 6, there was no significant correlation between rs3753841 (COL11A1) polymorphism and ocular biometry parameters $(p>0.05)$ in acute PACG. In this group, the homozygous mutant polymorphism was most frequent in shallow ACD, short AL, and thick lens.

In the chronic PACG group, we found a statistically significant correlation between genetic polymorphisms and ocular biometry, as shown in Table 7. This significant correlation of genetic mutation was found especially with ACD ( $p=0.009)$ and AL ( $p=0.01)$, but there was no correlation with LT $(\mathrm{p}=0.59)$. There was a tendency of different mutations between the two groups. While homozygous mutant was more common in acute PACG, heterozygous mutant was found to be more related with shallow ACD and short AL in chronic PACG.

\section{DISCUSSION}

There are several terminologies and classifications related to angleclosure glaucoma. In general, angle-closure glaucoma is divided into

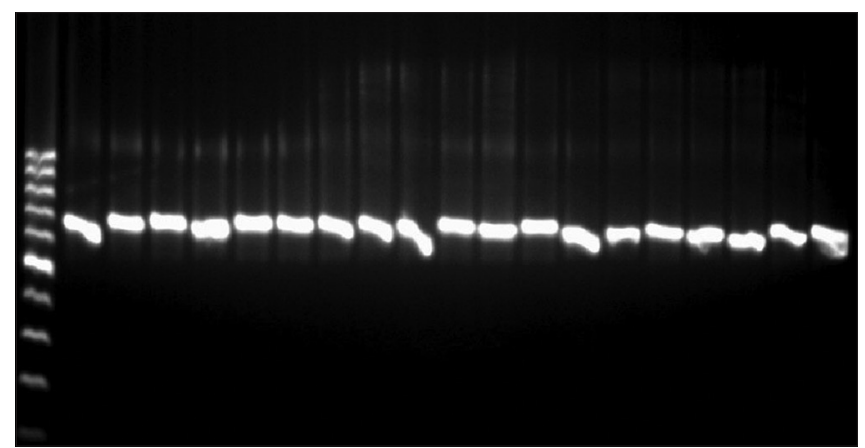

Fig. 1: Electrophoresis and DNA extraction results showing DNA bands from the isolated gene

Table 5: Locus polymorphisms of rs3753841 (COL11A1) in both groups

\begin{tabular}{|c|c|c|c|c|}
\hline \multirow[t]{2}{*}{ Polymorphism } & \multicolumn{2}{|l|}{ PACG } & \multirow{2}{*}{$\begin{array}{l}\text { Total } \\
\text { f (\%) }\end{array}$} & \multirow[t]{2}{*}{$\mathbf{p}$} \\
\hline & $\begin{array}{l}\text { Acute } \\
\text { f (\%) }\end{array}$ & $\begin{array}{l}\text { Chronic } \\
\text { f (\%) }\end{array}$ & & \\
\hline Wild type (CC) & (1) 4 & (3) 12 & (4) 8 & 0.009 \\
\hline Mutant homozygote (TT) & (14) 56 & (8) 32 & (22) 44 & \\
\hline Mutant heterozygote (CT) & (10) 40 & (14) 56 & (24) 48 & \\
\hline
\end{tabular}

PACG: Primary angle-closure glaucoma primary angle-closure suspect (PACS), primary angle-closure (PAC), and PACG. This classification is based on intraocular pressure (IOP), gonioscopy examination, optic nerve evaluation, and visual field defect. PACS is defined as the non-visibility of the trabecular meshwork for 180 degrees or more. In PACS, contact between peripheral iris and peripheral trabecular meshwork is present but not accompanied by PAS and increased IOP [7-9].

PAC indicated by shallow anterior chamber angle (ACA) and signs of trabecular meshwork obstruction by peripheral iris and absence of glaucomatous optic neuropathy during fundus examination. PACG, on the other hand, is indicated by conditions of PAC but followed by glaucomatous optic neuropathy finding. Clinical manifestation of PACG based on duration and onset is divided into acute PACG $(20-30 \%$ of cases) and chronic PACG (70-80\% of cases) $[7,8,10]$.

Acute PACG transpires when the entire trabecular meshwork is covered expeditiously by peripheral iris, causing a sudden increase in IOP and if not lowered immediately, this condition will cause damage to the intraocular structure, especially the optic nerve, which later can result in visual impairment. Otherwise, chronic PACG is indicated by the gradual movement of peripheral iris to cover trabecular meshwork and form of peripheral anterior synechiae (PAS). These synechiae can occur in certain effects only in a small quadrant at an early stage, but then it can gradually expand on the whole surface of the trabecular meshwork. This condition is usually asymptomatic because IOP gradually increases over a long period of time so that the eye has enough time to adjust to high IOP and finally all the axons in the optic nerve are damaged and a visual field defect arises $[7,8,10]$

In this study, we found the polymorphisms of rs3753841 (COL11A1) polymorphisms in both PACG groups. Nevertheless, there were different trends between the two groups; homozygous mutant was the most common type of mutation in acute PACG group while in chronic PACG group, heterozygous mutant was the most common type and this difference was statistically significant.

The COL11A1 gene is known to play a role in the production of Type $\mathrm{XI}$ collagen components, known as pro-alpha1 (XI) chains, which is one of the main components of the extracellular matrix. Extracellular matrix is one of the components of tissues involved in the path of the aqueous humor flow; therefore, the variation of this gene is thought to play a role in causing resistance to the flow of the aqueous humor and IOP elevation. In addition, an incremented regulation of COL11A1 gene mutation was found in glaucoma patients, which indicates the involvement and expression of extracellular matrix genes in the pathogenesis of glaucoma. The COL11A1 gene mutation was known to

Table 6: Correlation between polymorphisms and ocular biometry in acute PACG

\begin{tabular}{|c|c|c|c|c|c|c|c|c|c|}
\hline \multirow[t]{2}{*}{ Polymorphism locus rs3753841 } & \multicolumn{3}{|l|}{ ACD } & \multirow[t]{2}{*}{ p-value } & \multirow{2}{*}{$\frac{\mathrm{AL}}{<24}$} & \multirow[t]{2}{*}{ p-value } & \multicolumn{2}{|l|}{ LT } & \multirow[t]{2}{*}{ p-value } \\
\hline & $<2.91$ & $2.91-3.24$ & $>3.24$ & & & & $3.59-5.07$ & $>5.07$ & \\
\hline Wild type (CC) & 1 & 0 & 0 & 0.39 & 1 & 0.11 & 1 & 0 & 0.39 \\
\hline Mutant homozygote (TT) & 11 & 1 & 2 & & 14 & & 8 & 6 & \\
\hline Mutant heterozygote (CT) & 10 & 0 & 0 & & 10 & & 8 & 2 & \\
\hline Total & 22 & 1 & 2 & & 25 & & 17 & 8 & \\
\hline
\end{tabular}

ACD: Anterior chamber depth, AL: Axial length, LT: Lens thickness, PACG: Primary angle-closure glaucoma

Table 7: Correlation between polymorphisms and ocular biometry in chronic PACG

\begin{tabular}{|c|c|c|c|c|c|c|c|c|c|}
\hline \multirow[t]{2}{*}{ Polymorphism locus rs3753841 } & \multicolumn{3}{|l|}{ ACD } & \multirow[t]{2}{*}{ p-value } & \multirow{2}{*}{$\frac{\mathrm{AL}}{<24}$} & \multirow[t]{2}{*}{ p-value } & \multicolumn{2}{|l|}{ LT } & \multirow[t]{2}{*}{ p-value } \\
\hline & $<2.91$ & $2.91-3.24$ & $>3.24$ & & & & $3.59-5.07$ & $>5.07$ & \\
\hline Wild type (CC) & 3 & 0 & 0 & 0.01 & 3 & 0.00 & 3 & 0 & 0.59 \\
\hline Mutant homozygote (TT) & 6 & 2 & 0 & & 8 & & 6 & 0 & \\
\hline Mutant heterozygote (CT) & 14 & 0 & 0 & & 14 & & 14 & 2 & \\
\hline Total & 23 & 2 & 0 & & 25 & & 23 & 2 & \\
\hline
\end{tabular}

ACD: Anterior chamber depth, AL: Axial length, LT: Lens thickness, PACG: Primary angle-closure glaucoma 
play a role in Marshall syndrome and Type 2 Stickler syndrome, both of which have non-progressive axial myopia features $[4,7,11]$.

The COL11A1 gene additionally has the role of regulating the production of type XI collagen components, known as pro-alpha1 (XI) chains. This Type XI collagen can be found in tissues, such as the inner ear, vitreous, and pulposus nucleus, which is the middle part of the disk between the spines. Type XI collagen additionally avails maintaining distance and width of collagen Type II molecules which is an important component of vitreous and cartilage $[4,7]$

This study is the first study of gene polymorphisms in PACG subtype conducted in Southeast Asia. It is thought that there might be a possibility of ethnic factors having an important in polymorphisms of rs3753841 (COL11A1) in PACG. Based on ethnicity, a statistically significant correlation between COL11A1 polymorphisms and PACG was found in Australia ( $p=0.017)$ [5]. However, other studies in Nepal $(p=0.308)$, China $(p=0.054)$, and South India $(p=0.127)$ showed no significant relationship between these genetic polymorphisms and glaucoma $[5,6,12]$. Therefore, we can conclude that based on ethnicity, our study is the second study after the Australian population, which resulted a significant correlation between COL11A1 and PACG, especially in chronic subtypes.

Previous ocular biometric studies have shown that eyes with PACG have several distinct characteristics compared to normal eyes, such as a shallower anterior chamber, thicker lens, and shorter AL [3,13]. Eyes with PACG usually have anatomical features of the small cornea, shallow ACD, thick lens, anteriorly positioned lens, and short AL. Furthermore, some cases of angle-closure glaucoma are not fully explained by these anatomical risk factors. With the increasing use of new anterior chamber assessment techniques such as ultrasound bio-microscopy and optical coherence tomography, anterior segment parameters can be precisely measured [14-16]. To the best of our knowledge, variation in ocular biometric measurements between acute and chronic PACG has not been widely evaluated.

In this study, we classified PACG based on its clinical presentation, particularly the suddenness of the attacks, into acute and chronic PACG. Acute PACG is identified by rapid movement of iris covering the entire trabecular meshwork, causing a sudden and rapid symptomatic elevation of IOP. If this condition does not resolve spontaneously or immediately under medical intervention, it will get worse into an advanced chronic stage. Otherwise, in chronic PACG, the iris gradually covers the trabecular meshwork, leading to the formation of PAS.

The PAS is usually distinct or multi-centered at first, then gradually expand and fuse together, covering the trabecular meshwork in its entirety. $[7,8]$ Chronic PACG is often painless and asymptomatic because of gradual elevation of IOP in a long period of time; thus the eyes have the time to adapt to those changes in IOP. This classification can be used in determining the appropriate medical intervention for PACG based on its clinical presentation [7]

Previous ocular biometry studies have indicated that eyes with PACG usually present with different characteristics compared to those of normal eyes, such as shallow ACD, thicker LT, and shorter AL. When compared within angle-closure classification, the study by George et al. showed that there were no significant differences in ocular biometric parameters between patients with occludable angle and angle-closure glaucoma [3]. Low compared ocular biometry values in normal population versus eyes with PACG and indicated that shallow ACD in eyes with PACG was possibly caused by incoordination between the lens and AL. A thick lens with a more posterior position can cause rotation of the corpus ciliary, which can narrow down the ACA $[15,17]$. An eye with ACD of $2.5 \mathrm{~mm}$ or more was found to be less likely to develop into PACG. Another study in Nepal also found that AL was shorter in eyes with PACG than in the normal group $[15,18]$. Therefore, initial assessment of ocular biometric parameters can be used as a screening tool for patients with angle-closure glaucoma.
In this study, the ACD measurement was grouped into shallow $(<2.91 \mathrm{~mm})$, normal (2.91-3.24 mm), and deep ( $>3.24 \mathrm{~mm})$. AL value was classified into short $(<24 \mathrm{~mm})$, normal $(24-26 \mathrm{~mm})$, and long ( $>26 \mathrm{~mm})$, while LT measurement was divided into thin $(<3.59 \mathrm{~mm})$, normal $(3.59-$ 5.07), and thick ( $>5.07)$. There was a tendency for ACD to be deeper and for LT to be thicker than the normal values. Meanwhile, based on AL classification, all patients in both groups were in the short AL category. Nevertheless, there was no statistically significant difference in ocular biometry measurement between the two groups. Therefore, it is estimated that other trigger factors, such as poor lighting, might contribute to the development of acute or chronic PACG instead of its high-risk anatomical features of the anterior segment.

ACD is determined by various factors such as gender and race or ethnicity. The deepest ACD is within the central area which is normally around $3 \mathrm{~mm}$. Feng et al. conducted ACD evaluation in several countries representing all continents and estimated that the average ACD of the central area was $3.11 \mathrm{~mm}$, with a normal range of 2.91-3.24 mm [19]. When compared to that normal value, the mean ACD measurement in this study was below the normal range. Previously, Razeghinejad and Banifatemi evaluated ocular biometry in angle-closure and obtained an average ACD in acute PACG of $2.52 \pm 0.22 \mathrm{~mm}$ [3]

The mean AL measurement obtained in this study was $21.86 \pm 1.31 \mathrm{~mm}$ for acute PACG and 21.86 $\pm 1.31 \mathrm{~mm}$ for chronic PACG. Previous studies found that PACG patients had shorter AL than normal value. Thapa et al. compared average AL of occludable angle with the normal population and found mean AL of $22.8 \pm 0.83 \mathrm{~mm}$ in subjects with occludable angle, which was significant when compared to the normal population $(p<0.05)$ [15]. That result was slightly different from the average AL measurement obtained in our study.

Since all patients in both groups had short AL category, we then classified the short AL into more specific ranges; <21 mm, 21-22 mm, and $23-24 \mathrm{~mm}$. Based on that classification, we found a significant difference in both groups, in which acute PACG patients had shorter AL compared to chronic PACG patients. This result showed the possibilities of AL measurement having a more significant value in causing a crowded anterior segment, in line with the progression of PAC/PACG into the acute or chronic stage.

Razeghinejhad and Banifatemi found no significant difference in the average value of AL among PACS, PACG, and APAC ( $p=0.287)$, with the shortest AL were found in the APAC group. Similar to that study, even though we found no statistically significant difference in $\mathrm{AL}$ measurement between the two groups ( $p>0.05)$, yet we can conclude that both acute and chronic PACG patients had shorter AL compared to normal value. Furthermore, we found that the average AL measurement in acute PACG was shorter compared to those of chronic PACG. This result might suggest that eyes with shorter AL might have a higher risk of developing of PACG into the acute attack.

The lens is generally thicker in PACG than in normal eyes. This thicker lens is usually followed by a more anteriorly-positioned lens. Lowe estimated that thick crystalline lenses caused narrowing of the ACD up to $0.35 \mathrm{~mm}$, but more anteriorly-positioned lens could narrow down the ACD up to $0.65 \mathrm{~mm}$. The increase in LT value is also in line with age because of the lens growth with an increased number of lens fibers occurring continuously for a lifetime $[13,20]$. Razeghinejad and Banifatemi found that the mean LT in PACG was $4.54 \pm 0.47 \mathrm{~mm}$ [3]. The mean LT value in our study was not much different from previous studies. We obtained a mean LT of $5.01 \pm 0.49 \mathrm{~mm}$ in acute PACG and $4.91 \pm 0.42 \mathrm{~mm}$ in chronic PACG.

The correlation between polymorphisms of rs3753841 (COL11A1) and ocular biometry was also evaluated in both groups. In this study, a significant correlation was obtained between rs3753841 (COL11A1) polymorphisms and ocular biometry in chronic PACG. This genetic polymorphism was especially correlated with ACD $(p=0.009)$ and AL $(p=0.01)$, but no significant relationship was found with LT $(p=0.599)$. 
Heterozygous mutants were the most common mutations related to shallow ACD and short AL. In contrast to chronic PACG, the correlation between rs3753841 (COL11A1) polymorphisms with ocular biometry in acute PACG was not statistically significant ( $p>0.05)$.

Correlation between molecular genetics with ocular biometry parameters in angle-closure glaucoma has not been largely evaluated. One of the few studies that evaluated the correlation between ocular biometry and these genetic polymorphisms was the study by Shi et al., who found no significant correlation with ocular biometry in the PAC group, although these polymorphisms were detected in eyes with PACG [21]. Our results also supported a recent study by Wan et al. in China, which confirmed the role of COL11A1 polymorphisms as a potential gene for developing PACG and the possibility of these polymorphisms to be related to the course of PACG [11]

\section{CONCLUSION}

Based on our results, we confirmed the role of rs3753841 (COL11A1) polymorphisms in chronic PACG and its correlation with shallow ACD and short AL. Therefore, this study also showed that one of the possible mechanisms of slow course in angle-closure of chronic PACG might be affected by the genetic mutations. In addition, no significant correlation found between the rs3753841 (COL11A1) polymorphisms and ocular biometry in acute PACG can indicate the role of other factors, other than anatomical features, that are more dominantly affecting the course of the disease into an acute onset or sudden pupillary block leading to a sudden increase in IOP.

\section{ACKNOWLEDGMENT}

This paper was supported by the Biomedical Laboratory of Medical Faculty of Andalas University, West Sumatra - Indonesia.

\section{AUTHORS' CONTRIBUTIONS}

The concept and method of this study were initiated by Fitratul Ilahi, as well as collecting the data and writing this manuscript. Andika Prahasta and Rika Susanti interpreted the data, analyzed the result, and built discussion. Jamsari analyzed the source literature and applied the statistics.

\section{CONFLICTS OF INTEREST}

All the authors declared that they have no conflicts of interest to disclose in publishing this article.

\section{AUTHORS' FUNDING}

All of the works and funds needed in this study were supported by the authors themselves.

\section{REFERENCES}

1. Foster PJ, Oen FT, Machin D, Ng TP, Devereux JG, Johnson GJ, et al. The prevalence of glaucoma in Chinese residents of Singapore: A cross-sectional population survey of the Tanjong Pagar district. Arch Ophthalmol 2000;118:1105-11.

2. Ilahi F, Liyanti R. Quality of life assessment glaucoma patients based on glaucoma symptom scale and glaucoma quality of life-15 score at M. Djamil Hospital Padang. Ophthalmol Indones 2017;43:57-62.
3. Razeghinejad MR, Banifatemi M. Ocular biometry in angle closure. J Ophthalmic Vis Res 2013;8:17-24.

4. Vithana EN, Khor CC, Qiao C, Nongpiur ME, George R, Chen LJ, et al. Genome-wide association analyses identify three new susceptibility loci for primary angle closure glaucoma. Nat Genet 2013;44:1142-6.

5. Awadalla MS, Thapa SS, Hewitt AW, Burdon KP, Craig JE. Association of genetic variants with primary angle closure glaucoma in two different populations. PLoS One 2013;8:e67903.

6. Chen Y, Chen X, Wang L, Hughes G, Qian S, Sun X. Extended association study of PLEKHA7 and COL11A1 With primary angle closure glaucoma in a han Chinese population. Invest Ophthalmol Vis Sci 2014;55:3797-802.

7. Sun X, Dai Y, Chen Y, Yu D, Cringle SJ, Chen J, et al. Primary angle closure glaucoma: What we know and what we don't know. Prog Retin Eye Res 2016;46:26-45.

8. Stamper RL, Lieberman MF, Drake MV. Primary angle closure glaucoma. In: Becker-Shaffer's Diagnosis and Therapy of the Glaucomas. $8^{\text {th }}$ ed. USA: Elsevier; 2009. p. 196-214.

9. Skuta GL, Cantor LB, Cioffi GA. Angle-closure glaucoma. In: Glaucoma. San Fransisco: American Academy of Ophthalmology; 2014. p. 109-37.

10. National Health and Medical Research Council. Angle closure glaucoma. In: Guidelines for the Screening, Prognosis, Diagnosis, Management, and Prevention of Glaucoma. Canberra: National Health and Medical Research Council Guidelines; 2010. p. 44-5.

11. Wan Y, Li S, Gao Y, Tang L, Cao W, Sun X. COL11A1 polymorphisms are associated with primary angle-closure glaucoma severity. J Ophthalmol 2019;2019:2604386.

12. Duvesh R, Verma A, Venkatesh R, Kavitha S, Ramulu PY, Wojciechowski R, et al. Association study in a south indian population supports Rs1015213 as a risk factor for primary angle closure. Invest Ophthalmol Vis Sci 2013;54:5624-8.

13. Lowe RF. Aetiology of the anatomical basis for primary angle-closure glaucoma. Biometrical comparisons between normal eyes and eyes with primary angle-closure glaucoma. Br J Ophthalmol 1970;54:165-9.

14. Hamzeh N, Moghimi S, Latifi G, Mohammadi M, Khatibi N, Lin SC. Lens thickness assessment: Anterior segment optical coherence tomography versus A-scan ultrasonography. Int J Ophthalmol 2014;8:1151-5.

15. Thapa SS, Paudyal I, Khanal S, Paudel N, van Rens GH. Comparison of axial lengths in occludable angle and angle-closure glaucoma--the bhaktapur glaucoma study. Optom Vis Sci 2011;88:150-4.

16. George R, Paul PG, Baskaran M, Ramesh SV, Raju P, Arvind H, et al. Ocular biometry in occludable angles and angle closure glaucoma: A population based survey. Br J Ophthalmol 2003;87:399-402.

17. Bourne RR, Sørensen KE, Klauber A, Foster PJ, Johnson GJ, Alsbirk PH. Glaucoma in east greenlandic inuit--a population survey in ittoqqortoormiit (scoresbysund). Acta Ophthalmol Scand 2001;79:462-7.

18. Nolan WP, Baasanhu J, Undraa A, Uranchimeg D, Ganzorig S, Johnson GJ. Screening for primary angle closure in Mongolia: A randomised controlled trial to determine whether screening and prophylactic treatment will reduce the incidence of primary angle closure glaucoma in an East Asian population. $\mathrm{Br} \mathrm{J}$ Ophthalmol 2003;81:271-4.

19. Feng MT, Belin MW, Grewal SPS, Yan W, Shaheen MS, Mcghee C, et al. Anterior chamber depth in normal subjects by rotating scheimpflug imaging. Saudi J Ophthalmol 2011;25:255-9.

20. Tarongoy P, Ho CL, Walton DS. Angle-closure glaucoma: The role of the lens in the pathogenesis, prevention, and treatment. Surv Ophthalmol 2009;54:211-25.

21. Shi $\mathrm{H}$, Zhu R, Hu N, Shi J, Zhang J, Jiang L, et al. An extensive replication study on three new susceptibility loci of primary angle closure glaucoma in Han Chinese: Jiangsu Eye Study. J Ophthalmol 2013;2013:641596. 